Article

\title{
Establishing Correlations between Breast Tumor Response to Radio-Immunotherapy and Radiomics from Multi-Parametric Imaging: An Animal Study
}

\author{
Anis Ahmad ${ }^{1,+}{ }^{D}$, Tulasigeri M. Totiger ${ }^{1,+}$, Ana Paula Benaduce ${ }^{2}$, Brian Marples ${ }^{1,3}$ \\ and Ivaylo Bodganov Mihaylov ${ }^{1, *}$ \\ 1 Department of Radiation Oncology, Miller School of Medicine, Sylvester Comprehensive Cancer Center, \\ University of Miami, Miami, FL 33136, USA; axa1458@med.miami.edu (A.A.); \\ tmt78@med.miami.edu (T.M.T.); Brian_Marples@URMC.Rochester.edu (B.M.) \\ 2 Department of Biological Sciences, Florida International University, Miami, FL 33199, USA; \\ abenaduc@fiu.edu \\ 3 Department of Radiation Oncology, University of Rochester, 601 Elmwood Avenue, Box 647, \\ Rochester, NY 14642, USA \\ * Correspondence: i.mihaylov@med.miami.edu \\ + These authors contributed equally to this work.
}

Received: 23 July 2020; Accepted: 14 September 2020; Published: 17 September 2020

\begin{abstract}
Triple-negative breast cancer (TNBC), which is a type of invasive breast cancer, is characterized by severe disease progression, poor prognosis, high recurrence rate, and short survival. We sought to gain new insight into TNBC by applying computed tomography (CT) and magnetic resonance (MR) quantitative imaging (radiomics) approaches to predict the outcome of radio-immunotherapy treatments in a syngeneic subcutaneous murine breast tumor model. Five Athymic Nude mice were implanted with breast cancer cell lines (4T1) tumors on the right flank. The animals were CT- and MRI-imaged, tumors were contoured, and radiomics features were extracted. All animals were treated with radiotherapy (RT), followed by the administration of PD1 inhibitor. Approximately 10 days later, the animals were sacrificed, tumor volumes were measured, and histopathology evaluation was performed through Ki-67 staining. Linear regression modeling between radiomics and Ki-67 results was performed to establish a correlation between quantitative imaging and post-treatment histochemistry. There was no correlation between tumor volumes and Ki-67 values. Multiple CT- and MRI-derived features, however, correlated with histopathology with correlation coefficients greater than 0.8. MRI imaging helps in tumor delineation as well as an additional orthogonal imaging modality for quantitative imaging purposes. This is the first investigation correlating simultaneously CT- and MRI-derived radiomics to histopathology outcomes of combined radio-immunotherapy treatments in a preclinical setting applied to treatment naïve tumors. The findings indicate that imaging can guide discrimination between responding and non-responding tumors for the combined RT and ImT treatment regimen in TNBC.
\end{abstract}

Keywords: immunotherapy; CT; MRI; radiomics; radiotherapy; imaging; outcome; histochemistry

\section{Introduction}

Triple-negative breast cancer (TNBC) represents approximately $15-20 \%$ of all newly-diagnosed breast cancers, and it remains the most aggressive subtype with the poorest outcome [1]. No molecular targets exist for TNBC, and alternative treatment strategies are required. Radiotherapy (RT) is a standard-of-care treatment and RT-induced DNA damage causes direct tumor cell death. However, $\mathrm{RT}$ also induces immune responses through the release of tumor antigens and the generation of a 
favorable inflammatory cytokine [2]. Immune-checkpoint inhibitors (immunotherapy, or ImT) can augment RT effects by increasing tumor-specific T-cell proliferation, thereby improving outcomes over RT or ImT alone [3,4]. In preclinical studies the combination of RT and anti-PD-1/PD-L1 therapies activated CD8 T-cells, reduced inhibitory mechanisms, and induced abscopal effect [4-8].

Quantitative imaging (radiomics) comprises both semantic (volume, shape, etc.) and agnostic (texture and others) tumor features. Texture in particular, is a property commonly used for image classification in the field of pattern recognition. There are three different approaches used in image processing to find the textural feature of a region of interest in an image: first-order features, second-order features, and higher-order features. The first order features use statistical moments of the intensity histogram of the image and do not contain information about the relative position of pixels with respect to each other. Second order features employ the angular nearest-neighbor gray tone spatial-dependence matrices, also known as gray level co-occurrence matrices. Higher order features can be obtained by more complex manipulations of the image gray levels.

Textures have been used to define tumor response to treatment $[9,10]$. Recently, radiomics have been applied to patient cohorts treated with immunotherapy alone for metastatic non-small cell lung cancer and they were found helpful in prediction of response to therapy [11,12]. It has furthermore been demonstrated that when RT is incorporated in the predictive models as a covariate, it adds additional value to radiomics and ImT outcome correlations $[13,14]$. Other investigators have argued that the combination of $\operatorname{ImT}$ and RT has a synergistic effect on tumor response, surpassing the responses to $\operatorname{ImT}$ or RT alone $[15,16]$, which further supports the abovementioned findings.

The purpose of this work is to apply CT and MRI radiomics to combined radio-immunotherapy $(\operatorname{RImT})$ treatments, when applied to treatment naïve tumors, and determine if imaging better discriminates between responding and non-responding tumors. To our knowledge, this is the first work where tumor response to RImT regimen is assessed through histopathology evaluation.

\section{Materials and Methods}

\subsection{Preparation of Cell Suspension}

The $4 \mathrm{~T} 1$ breast cancer cell lines were cultured in RPMI-1640 medium (Gibco ${ }^{\circledR}$; Thermo Fisher Scientific, Inc., Waltham, MA, USA), supplemented with $10 \%$ fetal bovine serum (Gibco ${ }^{\circledR}$; Thermo Fisher Scientific Inc.) and 1\% penicillin-streptomycin (HyClone; GE Healthcare Life Sciences, Logan, UT, USA). For subcutaneous implantation, a cell suspension of a density of $1 \times 10^{4}$ cells $/ 25 \mu \mathrm{L}$ was prepared for each animal.

\subsection{Animal Model}

Female BALB/c mice ( $n=5 ; 8$ weeks old; $18-20 \mathrm{~g}$ ) were purchased from Jackson Laboratory (Bar Harbor, ME, USA) and were housed at $22 \pm 5^{\circ} \mathrm{C}$ in a $12 \mathrm{~h} \mathrm{light/dark} \mathrm{cycle} \mathrm{and} \mathrm{fed} \mathrm{rodent} \mathrm{chow}$ and water freely. Mice were subcutaneously inoculated with $25 \mu \mathrm{L} 4 \mathrm{~T} 1$ cell suspension $\left(1 \times 10^{4}\right)$ under $2 \%$ isoflurane. The skin was tented up, and the $4 \mathrm{~T} 1$ cells were implanted under the skin in the dorsal right flank regions. Tumor length (L) and width $(\mathrm{W})$ were measured every week using digital calipers. Tumor volume $(\mathrm{V})$ was calculated as $\left[\mathrm{V}=\left(\mathrm{L} \times \mathrm{W}^{2}\right) / 2\right]$. After tumor volumes reached $\sim 200 \mathrm{~mm}^{3}$, animals were CT- and MRI-imaged, and tumors were outlined as depicted in Figure 1. 


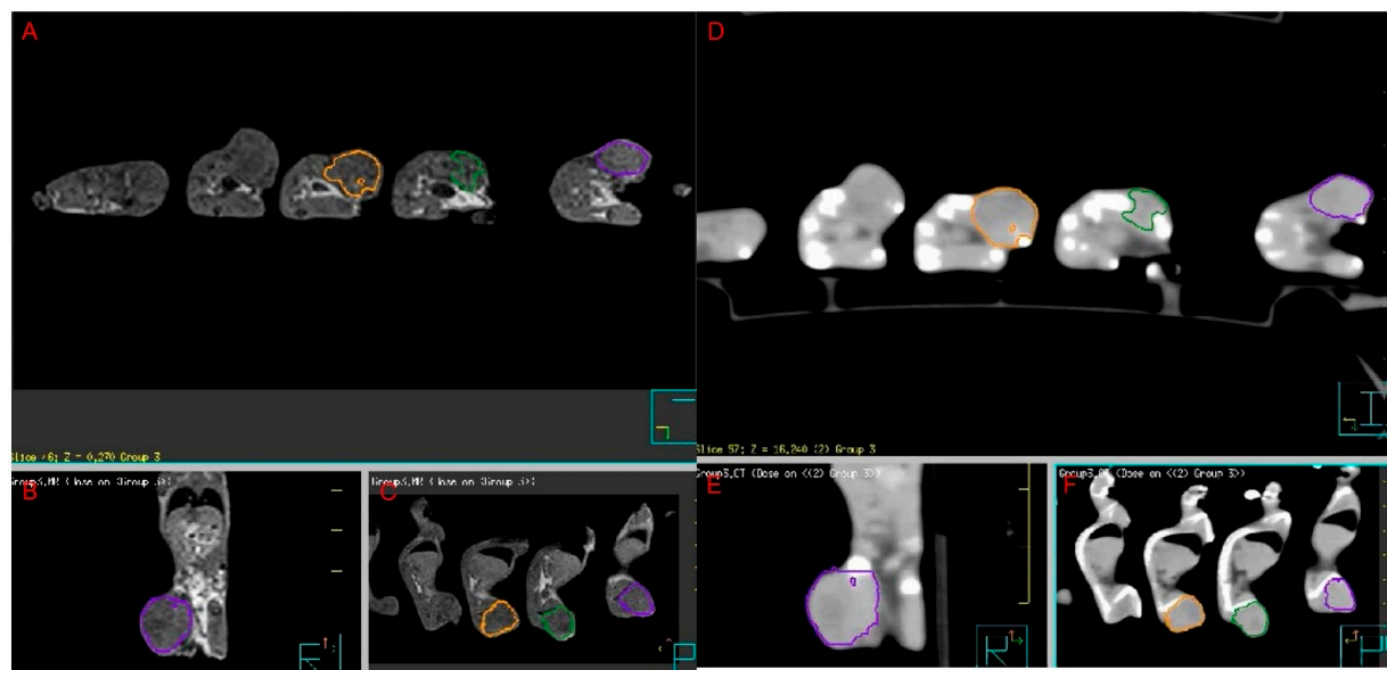

Figure 1. (A-C) T1 3T MRI image and (D-F) CT image of subcutaneously implanted breast tumors on mice right flanks.

All animal experiments were approved by the Institution of Animal Care and Use Committee of University of Miami.

\subsection{Imaging}

For the CT imaging studies, the image resolution was $0.4 \times 0.4 \times 0.6 \mathrm{~mm}^{3}$ (slice thickness $0.6 \mathrm{~mm}$ ), while for the T1-weighted MRI imaging studies, the resolution was $0.5 \times 0.5 \times 0.5 \mathrm{~mm}^{3}$ (slice thickness $0.5 \mathrm{~mm}$ ). The CT and MRI images of all five animals were acquired in a single scan (as depicted in Figure 1), thereby eliminating any inter-session scanner variability as well as voxel size variability. The flank tumors were contoured for each animal, and quantitative imaging features were extracted.

\subsection{Radiomics}

The radiomics features for both CT and MRI were extracted with our in-house software $[13,14,17]$, following published mathematical description [18]. The MRI radiomics in particular, were extracted after image intensity normalization [19-21], incorporated in the in-house software following a published procedure. Briefly, the intensity normalization followed a two-step approach: intensity scaling, followed by histogram normalization [21]. In the intensity scaling step, the histogram encompasses the entire range from low-intensity $(L I)$ to high-intensity $(H I)$ regions. The image intensities are mapped to the values between $L I$ and $H I$ domains according to:

$$
f^{\prime}(x, y, z)=\frac{f(x, y, z)-L I^{\prime}}{H I-L I}
$$

where $f(x, y, z)$ is gray value of original image at $(x, y, z)$, and $f^{\prime}(x, y, z)$ is the transformed gray-scale value. The $L I$ and $H I$ regions are defined as minimum and maximum deciles. During the histogram normalization step, the reference image histogram is stretched and shifted to cover gray levels from the input image according to:

$$
g^{i}(x, y, z)=\frac{H I-L I}{S_{\max }-S_{\min }}\left[g(x, y, z)-S_{\min }\right]+L I
$$

Where the histogram of the input image $g(x, y, z)$ starts at $S_{\min }$ and extends to $S_{\max }$ grayscale levels in the region of interest such that the normalized image $g^{\prime}(x, y, z)$ will lie between $L I$ and $H I$ values. Ninety-two (per imaging modality) geometric-, first-, second-, and third-order radiomics features for each tumor were obtained. For the first-, second-, and third-order radiomics, the imaging data 
was binned in 128 equally spaced bins, covering the entire gray-value range. The radiomics features were based on one dimensional (1D) histograms, 2D co-occurrence matrices (Matrix hereafter), 3D, and co-occurrence cubes (Cube hereafter). The Cube features are an extension of the Matrix features, where third-order joined probability is calculated, and the texture feature equations [18] are extended to 3D. The 3D features are derived from the tumor itself, where the gray values of the tumor voxels are normalized such that they are converted into probabilities. In turn, the Cube-type mathematical manipulations are applied to those probabilities, and 3D features are derived. The details of the used imaging features are described in detail in the Supplementary Materials. The 3D features refer to the extension of texture equations [18] to three dimensions, rather than the use of 3D (volumetric) imaging. All radiomics features were obtained from 3D imaging studies.

\subsection{Treatment}

After imaging, the animals were irradiated using a RadSource $2000 \mathrm{X}$-Ray Irradiator cabinet and organ-specific irradiation jigs ( $\left.160 \mathrm{kVp}, 25 \mathrm{~mA}, 0.5 \mathrm{mM} \mathrm{Cu}, 1.8 \mathrm{~Gy} \mathrm{~min}^{-1}\right)$ under $2 \%$ isoflurane in three equal-sized fractions to a total dose of 24 Gy over 3 consecutive days [15]. Following the irradiation, on 2 consecutive days, a single dose of PD-1 inhibitor was administered through intraperitoneal injection. When the apparent tumor volumes reached approximately $\sim 800 \mathrm{~mm}^{3}$, the animals were sacrificed.

\subsection{Histology}

Selected tumor tissues were dissected, paraffin-embedded, and sectioned ( $6 \mu \mathrm{m}$ thick) for histological examination. Sections were stained with Ki-67 staining according to the standard protocol at the histopathology core facility, University of Miami. Ki-67 staining images were visualized using a light microscope (VS120, Olympus, Tokyo, Japan) at 10× magnification. For the quantification of Ki-67 staining, images were analyzed using Image J software.

\subsection{Imaging Feature Selection}

The relations among the CT, MRI-derived radiomics and the Ki-67 values were established through linear regression modeling on a feature-by-feature basis. Furthermore, multiple regression analysis was also performed with the IBM SPSS Statistics V.25 (IBM Corp., Armonk, NY, USA) software package. Pearson's correlation coefficient was calculated to quantify the correlation between the radiomics features and the outcome as inferred based on the histopathology findings.

\section{Results}

\subsection{Describing the Correlation between the Tumor Volume and Ki-67}

The tumor volumes were measured by caliper and compared with Ki67 score (Figure 2).

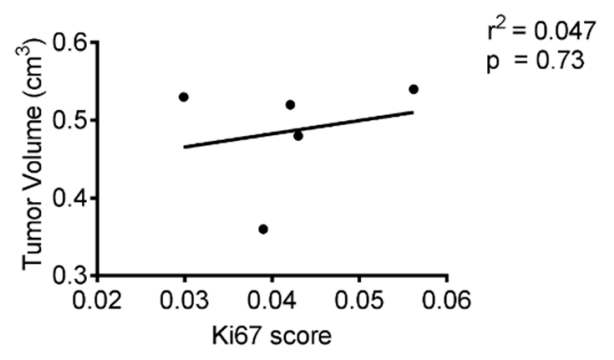

Figure 2. Comparison between tumor volume and Ki67 score.

These data indicate that there is no obvious correlation between tumor volumes and measured proliferating tumor cells. The estimated value for the Pearson correlation coefficient is 0.047, confirming the lack of linear dependence between tumor volume and Ki-67. At the same time, 
tumor volumes are surrogates for a response to treatment. The Ki67 staining score is strongly associated with cell proliferation, and it is used very commonly in routine histopathology (Figure 2).

\subsection{Correlation between Seven CT, Twenty-Four MRI Features and Ki-67 Score}

In the assessment of the correlation between the radiomics features and the Ki-67 values adjusted, $\mathrm{R}^{2}$ of more than 0.8 was considered as indicative of the linear correlation. Seven CT and $24 \mathrm{MRI}$ features exhibited a correlation with the post-treatment Ki-67 values according to this criterion (Figure 3).

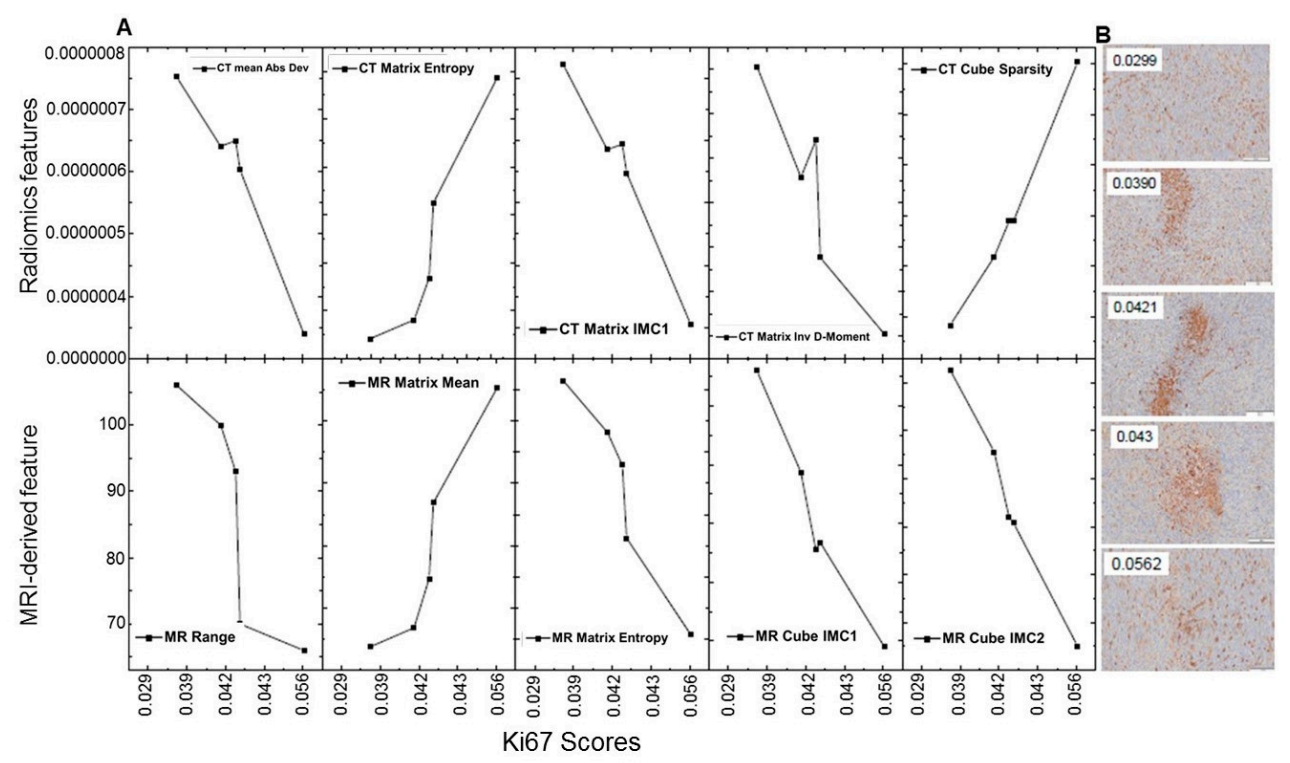

Figure 3. Dependence of five CT (A top row) and five MRI (A bottom row) radiomics features on post-treatment histochemistry proliferation marker Ki-67. The CT features (A top row) are mean absolute deviation 3D, cube IMC1, matrix IMC1, matrix inverse difference moment, and cube sparsity. The MRI features (bottom row) are mean absolute deviation 3D, matrix mean, matrix entropy, cube IMC1, and cube IMC2. Also, (B) the column on the right contains the histology slides with Ki-67 values denoted in the top left corner of each slide. The Ki-67 increases from top to bottom, as can be observed from the increasing amount of red-brown stain on the slides.

An example of five CT-derived radiomics features (Figure 3A top row) and five MRI-derived features (Figure 3A bottom row) as a function of post-treatment Ki-67 marker have been shown in Figure 3. Besides the imaging features, the figure displays the respective Ki-67 staining pictures with the denoted average Ki-67 values used in the assessment of post-treatment histopathology (Figure 3B).

The calculated correlation coefficients as well as the significance levels for the CT-derived features are outlined in Table 1. Figure 1 depicts only five features while the Table 1 presents details for all CT radiomics with $p$-value of $<0.05$. The Pearson correlation coefficients and the significance levels were calculated for the imaging features derived from the whole tumor. In order to further validate the presented results we generated two additional subregions in the tumors for both CT and MRI imaging modalities and we calculated the Pearson correlation coefficients for the radiomics derived from those subregions. Thereby, we were able to calculate the variance of in the Pearson correlation coefficients on feature-by-feature basis and they are outlined in the third column of the table. 
Table 1. Pearson correlation coefficients and significance level for CT-derived imaging features, which exhibit correlation with Ki-67marker.

\begin{tabular}{lccc}
\hline Feature & $\begin{array}{c}\text { Pearson } \\
\text { Correlation } \\
\text { Coefficient }\end{array}$ & Significance & $\begin{array}{c}\text { Variance of Pearson } \\
\text { Correlation } \\
\text { Coefficient }\end{array}$ \\
\hline Mean Abs Dev 3D & 0.94 & 0.007 & $2.4 \times 10^{-3}$ \\
Matrix IMC1 & 0.983 & 0.003 & $2.6 \times 10^{-3}$ \\
Matrix IMC2 & -0.893 & 0.041 & $2.8 \times 10^{-4}$ \\
Matrix Inverse Difference Moment Normalized & -0.926 & 0.024 & $9.4 \times 10^{-3}$ \\
Matrix Inverse Difference Normalized & -0.951 & 0.013 & $5.7 \times 10^{-2}$ \\
Cube Sparsity & 0.987 & 0.002 & $5.2 \times 10^{-2}$ \\
Cube IMC1 & 0.988 & 0.001 & $4.9 \times 10^{-3}$ \\
\hline
\end{tabular}

The correlation coefficients for the MRI-based radiomics are presented in Table 2. There are 24 MRI features with $p$-value of $<0.05$. Similarly to Table 1 above, the last column in Table 2 outlines the variance in the Pearson correlation coefficients where the two additional subregions in the tumor are used for radiomics extraction. The variances of Pearson correlation coefficients in both tables are very small, indicating that the observed relations are stable within the tumor volumes.

Table 2. Pearson correlation coefficients and significance level for MRI-based imaging features, which exhibit correlation with Ki-67marker.

\begin{tabular}{lccc}
\hline Feature & $\begin{array}{c}\text { Pearson } \\
\text { Correlation } \\
\text { Coefficient }\end{array}$ & Significance & $\begin{array}{c}\text { Variance of Pearson } \\
\text { Correlation } \\
\text { Coefficient }\end{array}$ \\
\hline Mean Abs Dev 2D & 0.977 & 0.004 & $8.6 \times 10^{-6}$ \\
Root Min Square 2D & 0.964 & 0.008 & $8.0 \times 10^{-5}$ \\
Entropy 2D & 0.971 & 0.006 & $6.3 \times 10^{-5}$ \\
Uniformity 2D & 0.964 & 0.008 & $7.6 \times 10^{-5}$ \\
Kurtosis 2D & 0.900 & 0.008 & $8.5 \times 10^{-5}$ \\
Skewness 2D & 0.922 & 0.026 & $6.7 \times 10^{-5}$ \\
Matrix Mean & 0.997 & 0.045 & $3.7 \times 10^{-4}$ \\
Matrix Variance & 0.932 & 0.021 & $4.1 \times 10^{-5}$ \\
Matrix Entropy & -0.960 & 0.01 & $4.9 \times 10^{-5}$ \\
Matrix Energy & 0.957 & 0.011 & $7.0 \times 10^{-5}$ \\
Matrix IMC1 & 0.967 & 0.007 & $1.2 \times 10^{-4}$ \\
Matrix IMC2 & -0.984 & 0.002 & $1.9 \times 10^{-4}$ \\
Matrix Cluster Prominence & -0.940 & 0.017 & $1.3 \times 10^{-4}$ \\
Matrix Cluster Contrast & -0.908 & 0.033 & $1.6 \times 10^{-5}$ \\
Matrix Cluster Tendency & -0.961 & 0.009 & $7.2 \times 10^{-5}$ \\
Matrix Cluster Homogeneity1 & -0.931 & 0.022 & $9.6 \times 10^{-6}$ \\
Matrix Cluster Homogeneity2 & -0.908 & 0.033 & $1.6 \times 10^{-5}$ \\
Cube Variance & 0.928 & 0.023 & $4.6 \times 10^{-3}$ \\
Cube Entropy & -0.954 & 0.012 & $1.8 \times 10^{-2}$ \\
Cube Energy & 0.956 & 0.011 & $6.9 \times 10^{-5}$ \\
Cube IMC1 & 0.974 & 0.005 & $4.8 \times 10^{-3}$ \\
Cube IMC2 & -0.991 & 0.001 & $2.5 \times 10^{-4}$ \\
Cube Cluster Prominence & -0.944 & 0.016 & $1.8 \times 10^{-3}$ \\
Cube Cluster Tendency & -0.961 & 0.009 & $1.7 \times 10^{-3}$ \\
\hline
\end{tabular}

For both CT and MRI radiomics, numerous imaging features demonstrate clear correlations with tumor cell proliferation markers derived from post-treatment histology as denoted in Tables 1 and 2. All of the correlation coefficients are at a significance level of $<0.05,11$ out of 24 are at a level of $<0.01$. The correlation coefficients in turn, are greater than 0.9 or less than -0.9 , indicating that imaging features can serve as a good method to predict Ki-67 proliferation marker in murine breast tumors. 
In addition to the univariate analyses presented above, multiple regression modeling was also employed for the CT and the MRI-derived imaging features. The multiple regression analyses for the CT-derived radiomics utilized all seven features denoted in Table 1. All of the features were set as independent variables, while the Ki-67 values were designated as a dependent variable. The regression method was selected to be "stepwise" in SPSS. The multiple regression model converged to a single variable-Cube IMC1. The resulting equation from the model is $(\mathrm{Ki}-67)_{\mathrm{CT}}=0.114+$ $0.145 \times(\text { Cube IMC1 })_{C T}$, with a correlation coefficient of 0.988 , and $p=0.001$. The $95 \%$ confidence interval for the slope and the intercept in the CT equation are 0.104 to 0.186 and 0.093 to 0.134 , respectively. Similarly, the multiple regression model for all MRI features from Table 2 resulted in a single predictor-Cube IMC2. The predictive linear equation is: $(K i-67)_{M R I}=0.221-0.22 \times(\text { Cube IMC2 })_{M R I}$ with a model $p$ of 0.001 . The $95 \%$ confidence interval for the slope and the intercept in the MRI equation are -0.275 to -0.168 and 0.178 to 0.265 , respectively. These results indicate that the third-order joint probability texture features, represented by the co-occurrence cubes, are best suited as predictors in the multiple regression analyses among imaging variables and post-therapy histopathology tumor proliferation markers in both CT and MRI.

\section{Discussion}

This work is the first demonstration of $\mathrm{CT}$ and MRI quantitative imaging application combined to radio-immunotherapy treatments in the treatment of naïve breast tumors, where the outcome is evaluated by histopathology. The use of a syngeneic animal model allows correlation with the actual number of surviving tumor cells rather than a surrogate for treatment response such as tumor volume. This critical point is illustrated by our findings that tumor volumes do not correlate with Ki-67 proliferation marker.

The superior MRI soft tissue contrast allows better delineation of tumors when fused to CT imaging studies. MRI results corroborated with the CT results, as opposed to current clinical radiomics publications on immunotherapy, where only CT-based features were utilized [11-14]. Besides the improved visualization capabilities, the presented results indicate that more radiomics features from the MRI studies correlate with the post-treatment immunohistochemistry in comparison to the CT-derived radiomics features from the same tumors. Therefore, MRI complements CT not only for visualization purposes, but also for quantitative imaging investigations. The MRI imaging of the five animals was performed in two consecutive sessions, each of approximately $\sim 30 \mathrm{~min}$. The CT imaging followed immediately after the MRI image acquisition. The same hardware (scanners) with the same imaging protocols were used for both MRI and CT imaging of the two batches of animals. Therefore, the variability of the imaging features due to the acquisition technique and the processing equipment is very minimal.

Since subcutaneous tumors were derived from the same cell line, the observed variations in the imaging features cannot be attributed to tumor genotype, which existence affects treatment outcomes in clinical studies. Instead, the variations in the radiomics features presented herein depict the changes in the tumor phenotype and in particular the tumor heterogeneity, which are probably due to the effects of the tumor microenvironment as well as the specific host traits [22].

\section{Conclusions}

Our study is the first investigation correlating CT and MRI-derived radiomics to histopathology outcomes of combined radio-immunotherapy treatments of treatment naïve breast tumors in a preclinical setting. The establishment of a predictive model for the histochemistry response based on radiomics will open a whole new paradigm in the radio-immunotherapy research and clinical applications. Arguably, the combination of radiotherapy and immunotherapy is an appealing treatment paradigm, and it is not unreasonable to hypothesize that in the near future, it will become the primary treatment modality for cancer. 
Supplementary Materials: The following are available online at http://www.mdpi.com/2076-3417/10/18/6493/s1. Definition of imaging features is shown in Supplementary Materials.

Author Contributions: Conceptualization, A.A., B.M. and I.B.M.; methodology, A.A., T.M.T., A.P.B., B.M. and I.B.M.; software, A.A., B.M. and I.B.M.; validation, A.A., T.M.T., A.P.B., B.M. and I.B.M.; formal analysis, A.A., T.M.T., A.P.B., B.M. and I.B.M.; validation, A.A., T.M.T., A.P.B., B.M. and I.B.M.; investigation, A.A., T.M.T., A.P.B., B.M. and I.B.M.; resources, A.A., T.M.T., A.P.B., B.M. and I.B.M.; data curation, A.A., T.M.T., B.M. and I.B.M.; writing-original draft preparation, A.A., T.M.T., B.M. and I.B.M.; writing-review and editing, A.A., B.M. and I.B.M.; visualization, A.A., T.M.T., A.P.B., B.M. and I.B.M.; supervision, A.A., B.M. and I.B.M.; project administration, A.A., B.M. and I.B.M.; funding acquisition, B.M. and I.B.M. All authors have read and agreed to the published version of the manuscript.

Funding: This work is funded in part by a Flight Attendant Medical Research Institute grant.

Acknowledgments: The authors acknowledge the Flight Attendant Medical Research Institute for the financial support.

Conflicts of Interest: The authors declare no conflict of interest.

\section{References}

1. Stagg, J.; Allard, B. Immunotherapeutic approaches in triple-negative breast cancer: Latest research and clinical prospects. Ther. Adv. Med. Oncol. 2013, 5, 169-181. [CrossRef] [PubMed]

2. Eriksson, D.; Stigbrand, T. Radiation-induced cell death mechanisms. Tumour Biol. 2010, 31, $363-372$. [CrossRef] [PubMed]

3. Park, W.; Kwon, D.; Saravia, D.; Desai, A.; Vargas, F.; El Dinali, M.; Warsch, J.; Elias, R.; Chae, Y.K.; Kim, D.W.; et al. Developing a Predictive Model for Clinical Outcomes of Advanced Non-Small Cell Lung Cancer Patients Treated With Nivolumab. Clin. Lung Cancer 2018, 19, 280-288.e284. [CrossRef] [PubMed]

4. Sharabi, A.B.; Nirschl, C.J.; Kochel, C.M.; Nirschl, T.R.; Francica, B.J.; Velarde, E.; Deweese, T.L.; Drake, C.G. Stereotactic Radiation Therapy Augments Antigen-Specific PD-1-Mediated Antitumor Immune Responses via Cross-Presentation of Tumor Antigen. Cancer Immunol. Res. 2015, 3, 345-355. [CrossRef]

5. Dovedi, S.J.; Adlard, A.L.; Lipowska-Bhalla, G.; McKenna, C.; Jones, S.; Cheadle, E.J.; Stratford, I.J.; Poon, E.; Morrow, M.; Stewart, R.; et al. Acquired resistance to fractionated radiotherapy can be overcome by concurrent PD-L1 blockade. Cancer Res. 2014, 74, 5458-5468. [CrossRef]

6. Hu, Z.S.I.; McArthur, H.L.; Ho, A.Y. The Abscopal Effect of Radiation Therapy: What Is It and How Can We Use It in Breast Cancer? Curr. Breast Cancer Rep. 2017, 9, 45-51. [CrossRef]

7. Liu, Y.; Dong, Y.P.; Kong, L.; Shi, F.; Zhu, H.; Yu, J.M. Abscopal effect of radiotherapy combined with immune checkpoint inhibitors. J. Hematol. Oncol. 2018, 11, 104. [CrossRef]

8. Ngwa, W.; Irabor, O.C.; Schoenfeld, J.D.; Hesser, J.; Demaria, S.; Formenti, S.C. Using immunotherapy to boost the abscopal effect. Nat. Rev. Cancer 2018, 18, 313-322. [CrossRef]

9. Gillies, R.J.; Kinahan, P.E.; Hricak, H. Radiomics: Images Are More than Pictures, They Are Data. Radiology 2016, 278, 563-577. [CrossRef]

10. Lambin, P.; Rios-Velazquez, E.; Leijenaar, R.; Carvalho, S.; van Stiphout, R.G.P.M.; Granton, P.; Zegers, C.M.L.; Gillies, R.; Boellard, R.; Dekker, A.; et al. Radiomics: Extracting more information from medical images using advanced feature analysis. Eur. J. Cancer 2012, 48, 441-446. [CrossRef]

11. Trebeschi, S.; Drago, S.G.; Birkbak, N.J.; Kurilova, I.; Calin, A.M.; Pizzi, A.D.; Lalezari, F.; Lambregts, D.M.J.; Rohaan, M.; Parmar, C.; et al. Predicting Response to Cancer Immunotherapy using Non-invasive Radiomic Biomarkers. Ann. Oncol. 2019, 30, 998-1004. [CrossRef] [PubMed]

12. Tunali, I.; Gray, J.E.; Qi, J.; Abdalah, M.; Jeong, D.K.; Guvenis, A.; Gillies, R.J.; Schabath, M.B. Novel clinical and radiomic predictors of rapid disease progression phenotypes among lung cancer patients treated with immunotherapy: An early report. Lung Cancer 2019, 129, 75-79. [CrossRef] [PubMed]

13. Spieler, B.; Azzam, G.; Asher, D.; Lopes, G.; Saravia, D.; Kwon, D.; Yechieli, R.; Dal Pra, A.; Diwanji, T.; Mihaylov, I.B. Overall Survival of Patients with Advanced NSCLC Treated with Nivolumab Correlates with Texture Features on Pre-Immunotherapy CT Imaging and Radiotherapy History. Int. J. Radiat. Oncol. Biol. Phys. 2019, 105, E531-E532. [CrossRef]

14. Spieler, B.O.; Saravia, D.; Lopes, G.; Azzam, G.; Kwon, D.; Dal Pra, A.; Yechieli, R.; Diwanji, T.; Mihaylov, I. Can texture analysis of pre-immunotherapy CT imaging predict clinical outcomes for patients with advanced NSCLC treated with Nivolumab? J. Clin. Oncol. 2019, 37, e20720. [CrossRef] 
15. Demaria, S.; Golden, E.B.; Formenti, S.C. Role of Local Radiation Therapy in Cancer Immunotherapy. JAMA Oncol. 2015, 1, 1325-1332. [CrossRef]

16. Reynders, K.; Illidge, T.; Siva, S.; Chang, J.Y.; De Ruysscher, D. The abscopal effect of local radiotherapy: Using immunotherapy to make a rare event clinically relevant. Cancer Treat. Rev. 2015, 41, 503-510. [CrossRef]

17. Padgett, K.; Mihaylov, I. Radiomics Feature Variability On 0.35T MR-Guided-RT System. Med. Phys. 2017, 44, 3148.

18. Leijenaar, R.T.; Carvalho, S.; Velazquez, E.R.; van Elmpt, W.J.; Parmar, C.; Hoekstra, O.S.; Hoekstra, C.J.; Boellaard, R.; Dekker, A.L.; Gillies, R.J.; et al. Stability of FDG-PET Radiomics features: An integrated analysis of test-retest and inter-observer variability. Acta Oncol. 2013, 52, 1391-1397. [CrossRef]

19. Do, Q.N.; Lewis, M.A.; Madhuranthakam, A.J.; Xi, Y.; Bailey, A.A.; Lenkinski, R.E.; Twickler, D.M. Texture analysis of magnetic resonance images of the human placenta throughout gestation: A feasibility study. PLoS ONE 2019, 14, e0211060. [CrossRef]

20. Loizou, C.P.; Pantziaris, M.; Pattichis, C.S.; Seimenis, I. Brain MR image normalization in texture analysis of multiple sclerosis. J. Biomed. Graph. Comput. 2012, 3. [CrossRef]

21. Sun, X.F.; Shi, L.; Luo, Y.S.; Yang, W.; Li, H.P.; Liang, P.P.; Li, K.C.; Mok, V.C.T.; Chu, W.C.W.; Wang, D.F. Histogram-based normalization technique on human brain magnetic resonance images from different acquisitions. Biomed. Eng. Online 2015, 14, 73. [CrossRef] [PubMed]

22. Anderson, A.R.; Weaver, A.M.; Cummings, P.T.; Quaranta, V. Tumor morphology and phenotypic evolution driven by selective pressure from the microenvironment. Cell 2006, 127, 905-915. [CrossRef] [PubMed]

(C) 2020 by the authors. Licensee MDPI, Basel, Switzerland. This article is an open access article distributed under the terms and conditions of the Creative Commons Attribution (CC BY) license (http://creativecommons.org/licenses/by/4.0/). 